\title{
Contaminación con Huevos de Toxocara sp y Evaluación Sanitaria de Parques en la Ciudad de Abancay, Perú
}

\author{
Contamination with Toxocara Sp Eggs and Health Assessment of Parks \\ in the City of Abancay, Peru \\ Cori Milagros Cáceres Pintoㅁ, Renzo Hernán Bustinza Cárdenas ${ }^{2}$, \\ Aldo Alim Valderrama Pomé $e^{3,4}$
}

\section{Resumen}

El objetivo de esta investigación fue determinar la asociación entre la contaminación con Toxocara sp y la clasificación sanitaria de parques de la Dirección de Higiene Alimentaria y Zoonosis, Ministerio de Salud (Perú), en la ciudad de Abancay, Perú. La investigación fue de tipo analítico, nivel básico y corte transversal. La toma de muestras $(n=276)$ se realizó en los 21 parques de la ciudad mediante un muestreo aleatorio sistemático a través de la metodología de la doble «W». La clasificación de los parques se realizó utilizando la ficha de evaluación del Programa de Vigilancia Sanitaria de Parques, valorando la infraestructura adecuada, ambiente y riesgo sanitario. El $66.7 \%$ de los parques se encontraron contaminados con Toxocara sp, y la frecuencia de contaminación con el parásito en las muestras fue de $26.8 \%$ (IC95\% = 21.4-32.2). Ningún parque calificó como amigable, $38.1 \%$ fueron poco amigables y $61.9 \%$ fueron considerados como no amigables. La ficha de evaluación del Programa de Vigilancia Sanitaria de parques no es aplicable para estimar la contaminación con Toxocara sp.

Palabras clave: contaminación, Toxocara, vigilancia sanitaria, parques

\section{AbSTRaCT}

The aim of this study was to determine the association between contamination with Toxocara sp and the sanitary classification of parks of the Department of Food Hygiene and Zoonosis, Ministry of Health (Peru), in the city of Abancay, Peru. The research was

\footnotetext{
${ }^{1}$ Servicio Nacional de Sanidad Agraria, Apurímac, Perú

${ }^{2}$ Municipalidad Distrital de Tamburco, Apurímac, Perú

${ }^{3}$ Facultad de Medicina Veterinaria y Zootecnia, Universidad Nacional Micaela Bastidas de Apurímac, Apurimac, Perú

${ }^{4}$ E-mail: alimvalderrama@gmail.com
}

Recibido: 19 de mayo de 2016

Aceptado para publicación: 30 de noviembre de 2016 
analytical, basic level and cross-sectional. Sampling $(\mathrm{n}=276)$ was conducted in all 21 city parks through a systematic random sampling using the double «W» methodology. The classification of parks was carried out using the assessment sheet of the Park Sanitary Surveillance Programme, assessing the infrastructure, environment, and health risks. The results showed that $66.7 \%$ of parks were contaminated with Toxocara sp and the frequency of samples contaminated with the parasite was $26.8 \%(95 \% \mathrm{CI}=21.4-32.2)$. None of the parks qualified as friendly, $38.1 \%$ were considered less friendly and $61.9 \%$ as not friendly. The evaluation form of the Park Sanitary Surveillance Programme was not applicable to estimate the contamination with Toxocara sp.

Key words: pollution, Toxocara, health surveillance, parks

\section{INTRODUCCIÓN}

La toxocariosis es una de las zoonosis más prevalentes a nivel mundial (Maguiña, 2010; Sariego et al., 2012), causada por el nematodo del género Toxocara (Chávez et al., 2002). Las especies Toxocara canis y Toxocara cati (parásitos intestinales de perros y gatos, respectivamente) son importantes para el ser humano. La hembra adulta produce hasta 200000 huevos al día, que son excretados en las heces de los animales infectados, depositándose en la tierra, donde se convierten en la forma infectante del huevo embrionado (Maguiña, 2010). Una infección ligera con $T$. canis produce 10000 huevos por gramo de heces, lo que implica que cada perro contribuya diariamente a la contaminación ambiental con casi 1.4 millones de huevos (OPS, 2003).

La principal fuente de infección es el suelo contaminado con huevos fértiles larvados del parásito, que pueden persistir infectantes por muchos años en suelo húmedo, fresco y sombrío, y a temperatura templada; no obstante, también soportan la desecación, pues presentan una cubierta muy resistente (Huapaya et al., 2009). Esta situación se produce como consecuencia de la defecación indiscriminada en estos sitios por perros y gatos infectados; de allí que no se debe permitir que los perros deambulen libremente por parques públicos. Los dueños deben recoger las heces de sus mascotas y desecharlas en los contenedores de basura biológica (Breña et al., 2011; Delgado y Rodríguez, 2009; OPS, 2003).

El humano adquiere la enfermedad a través del contagio vía oral, por agua, alimentos o manos contaminadas con huevos de este nematodo. Los niños tienen más contacto con el suelo y suelen practicar geofagia, de allí que están más expuestos y exhiben prevalencias más altas (OPS, 2003). La infección en personas es accidental y los parásitos en el cuerpo humano no pueden completar su maduración. Debido a esto, las larvas que ingresan al cuerpo migran durante meses por diversos órganos, ocasionando reacción inflamatoria local y sistémica, según el órgano afectado. Se describe que las larvas pueden sobrevivir por varios años en un hospedero humano, e incluso de por vida, causando hemorragia, necrosis, reacción inflamatoria eosinofilica y, eventualmente, la formación de granulomas (Delgado y Rodríguez, 2009; Huapaya et al., 2009; Sariego et al., 2012).

La contaminación de parques por huevos de Toxocara a nivel mundial oscila entre 2.9 a 75\% (Maguiña, 2010), y en el Perú se reportan prevalencias de 24 a 100\% (Castillo et al., 2001; Chávez et al., 2002; López et al., 2005; Young et al., 2011; Iannacone et al., 2012). 
La Dirección de Higiene Alimentaria y Zoonosis, órgano de línea de la Dirección General de Salud Ambiental del Ministerio de Salud de Perú, dentro del Programa de Vigilancia Sanitaria de Parques, cuenta con una ficha nacional de evaluación de parques amigables (DHAZ, 2016), la cual podría ser usada concomitantemente para estimar la presencia de Toxocara. Es así que el objetivo de esta investigación fue determinar la asociación entre la contaminación con Toxocara sp y la clasificación sanitaria de parques en la ciudad de Abancay, Perú.

\section{Materiales y Métodos}

El estudio se realizó en el distrito de Abancay, provincia de Abancay, Perú, durante los meses de junio a octubre de 2012 . La ciudad se sitúa a 2377 metros sobre el nivel del mar, en la vertiente oriental de los Andes y cuenta con una superficie de $313.1 \mathrm{~km}^{2}$ (INEI, 2016). El clima es mayormente templado, con temperaturas promedio de $18^{\circ} \mathrm{C}$, con valores de $67 \%$ de humedad relativa en los meses de febrero y marzo y $57 \%$ de humedad relativa en los meses de junio y julio (MPA, 2011). La investigación fue de tipo analítico, de nivel básico y de corte transversal.

El estudio comprendió las 21 plazas y parques de la ciudad, que fueron identificados mediante los mapas contemplados en el Plan de Desarrollo Urbano de la Municipalidad Provincial de Abancay (MPA, 2011). E1 muestreo contó con la autorización de la Municipalidad Provincial de Abancay, bajo la supervisión de la Gerencia de Medio Ambiente y Servicios Públicos.

Para la toma de muestras se aplicó el muestreo aleatorio sistemático a través de la metodología de la doble «W», trazando dos «W» opuestas entre sí en el área a muestrear. Se determina la longitud de cada «W» en pasos y se estima en forma proporcional el número de puntos de muestreo $(10 \%$ de la longitud), por lo que el número de muestras difiere de acuerdo al área de la plaza o parque. Las muestras, consistentes en tierra y pasto, se tomaron en un área de $5 \mathrm{~cm}$ de diámetro y $2.5 \mathrm{~cm}$ de profundidad (aproximadamente 2 a $5 \mathrm{~kg}$ por muestra) (DHAZ, 2016). Se obtuvieron 276 muestras que fueron depositadas en bolsas autosellantes de polietileno, debidamente identificadas con el nombre de la plaza o parque, número de muestra, fecha y hora. Las muestras se conservaron en cadena de frío a $7{ }^{\circ} \mathrm{C}$, hasta su envío al laboratorio (cada tres días, aproximadamente).

El análisis de las muestras se realizó en el Laboratorio Veterinario del Sur (LABVETSUR) de la ciudad de Arequipa. Se utilizó la técnica de flotación desarrollada por Willis, donde se emplea una solución acuosa saturada de cloruro de sodio (Cruz y Camargo, 2001).

La ficha de evaluación del Programa de Vigilancia Sanitaria de Parques de la Dirección de Higiene Alimentaria y Zoonosis se basa en la observación de la infraestructura, ambiente y riesgo sanitario, otorgando puntos en cada uno de los ítems, donde el total del puntaje categoriza a las plazas y parques como no amigables $(0-42$ puntos; $<50 \%)$, poco amigables (43-64 puntos; 50-75\%) y amigables (65-84 puntos; 75-100\%) (DHAZ, 2016).

Los datos fueron analizados mediante estadística descriptiva y con el Chi-cuadrado de Pearson e intervalos de confianza con un $\alpha=0.05$, utilizando el paquete estadístico SPSS v. 11.5.

\section{Resultados y Discusión}

\section{Frecuencia de Toxocara sp en Plazas y Parques}

Catorce de los 21 plazas y parques estuvieron contaminados con Toxocara sp, significando una prevalencia de $66.7 \%$. Asimismo, 26.8\% (IC95\% = 21.4-32.2\%) de las muestras de pasto y tierra estuvieron contaminadas con huevos de Toxocara sp (Cuadro 1). 
Cuadro 1. Frecuencia de Toxocara sp en 21 plazas y parques de la ciudad de Abancay, Perú (2012)

\begin{tabular}{lcc}
\hline & \multicolumn{2}{c}{ Muestras mixtas de tierra y pasto } \\
\cline { 2 - 3 } Plazas y parques & $\begin{array}{c}\text { Recolectadas } \\
(\mathrm{n})\end{array}$ & $\begin{array}{c}\text { Positivas a Toxocara sp } \\
(\%)\end{array}$ \\
\hline Parque Villa Gloria & 8 & 87.5 \\
Parque San Martín & 10 & 80.0 \\
Parque Daniel Alcides Carrión & 8 & 75.0 \\
Parque Imperial & 22 & 63.6 \\
Parque Santa Rosa & 8 & 62.5 \\
Parque Santa Martha & 12 & 58.3 \\
Parque Los Chancas & 8 & 50.0 \\
Parque Juan Pablo & 12 & 33.3 \\
Parque Francisco Bolognesi & 14 & 28.6 \\
Parque José María Arguedas & 12 & 25.0 \\
Plaza Micaela Bastidas & 22 & 22.7 \\
Parque Centenario & 16 & 18.7 \\
Plaza de Armas & 12 & 16.7 \\
Parque CER Taraccasa & & 6.7 \\
Parque Ingenieros & 30 & 0 \\
Parque de la Urb. Santa Rosa & 8 & 0 \\
Parque Señor de la Caída & 6 & 0 \\
Parque del Periodista & 18 & 0 \\
Plaza Centenario & 8 & 0 \\
Parque Ovalo el Olivo & 18 & 0 \\
Parque Piquichas & 876 & 0 \\
\hline Total & & 0.8 \\
\hline
\end{tabular}

${ }^{1}$ Parque Centro Ecológico Recreacional Taraccasa

${ }^{2}$ Intervalo de confianza al 95\%: 21.4 - 32.2\%

La prevalencia encontrada es superior a las reportadas en otras ciudades del país, como Ica con $52.5 \%$, Cusco con $32.6 \%$ (Iannacone et al., 2012) y Puno con $25 \%$ (Yapuchura, 2004; Iannacone et al., 2012); así como en los distritos de los conos Norte, Sur y Este de Lima con 29.6-41.1\% (Serrano et al., 2000; Chávez et al., 2002), Breña con $48 \%$ (Young et al., 2011) y la Provincia
Constitucional del Callao con 37.2\% (Chávez et al., 2002). Sin embargo, los resultados son similares a lo encontrado en el Cono Oeste de Lima con 63\% (Chávez et al., 2002; López et al., 2005) y Santiago de Surco con $69.2 \%$ (Iannacone et al., 2012). Por otro lado, existen pocas ciudades en el Perú que presentan prevalencias mayores a lo encontrado en este estudio, tales como Ferreñafe con $100 \%$, 
Huánuco con 75\% (Iannacone et al., 2012) y San Juan de Lurigancho con $70.6 \%$ (Castillo et al., 2001).

En el continente americano, la mayoría de países reportan prevalencias inferiores a las del presente estudio, tales como 31-63\% en Venezuela (Cazorla et al., 2007; Devera et al., 2015; Gallardo y Forlano, 2015), 12$63 \%$ en México (Tinoco et al., 2007; Romero et al., 2009, 2011, 2013), 52.9\% en Uruguay (Hernández et al., 2003), 53\% en Paraguay (Canese et al., 2003), 4-42\% en Colombia (Polo et al., 2007; Cala et al., 2010; Díaz et al., 2015), 63\% en Ecuador (Latorre y Nápoles, 2014), 63\% en Bolivia (Loza et al., 2006) y 7\% en Costa Rica (Paquet et al., 2007). Por otro lado, son pocos los reportes con prevalencias similares o superiores, tales como 10-67\% en Chile (Gutiérrez, 2004; Delgado y Rodríguez, 2009; Armstrong et al., 2011), 44-100\% en Cuba (Laird et al., 2000; Sariego et al., 2012) y $12-100 \%$ en Brazil (Lettieri et al., 2008; Santarém et al., 2008; Tiyo et al., 2008; Delgado y Rodríguez, 2009; Barbosa, 2010; Gallina et al., 2011; Alvares et al., 2012; Manini et al., 2012; Marques et al., 2012).

La prevalencia de Toxocara sp fue elevada, encontrándose por encima del promedio nacional que es de $52.5 \%$ (Iannacone $e t$ al., 2012). Así mismo, la condición de parques negativos a Toxocara no indica que esta sea su condición real, sino que la cantidad relativa de huevos es menor al límite inferior de detección de los métodos utilizados (Canese et al., 2003).

Los parques más contaminados con Toxocara sp son los que cuentan con mayor cuidado municipal y mayores áreas verdes sombreadas. Por el contrario, los parques con escasa o nula contaminación tienen tierras removidas, mayor área de cemento y cerco perimétrico (Chávez et al., 2002).

Además, se reporta que los parques públicos con más de $1000 \mathrm{~m}^{2}$ presentan mayores niveles de contaminación (Iannacone et al., 2012); sin embargo, el único parque que tiene estas dimensiones en la ciudad de Abancay es el Parque Centro Ecológico Recreacional Taraccasa que, no obstante, presentó una de las más bajas frecuencias de contaminación (6.7\%).

\section{Evaluación Sanitaria de Parques}

La evaluación sanitaria evidenció que ningún parque calificó como amigable, en tanto que ocho parques $(38.1 \%$ ) resultaron poco amigables y 16 (61.9\%) fueron no amigables. Los parques que calificaron como poco amigables fueron Santa Martha, Francisco Bolognesi, Micaela Bastidas, Plaza de Armas, Señor de la Caída, Periodista y Centenario, con 43 puntos o más (Figura 1).

Los parques en mejor estado de conservación suelen ser los más contaminados debido a que la estructura y composición del suelo juegan un papel muy importante, ya que la vegetación mantiene condiciones suficientes de humedad y microclimas favorables para el desarrollo de los huevos de Toxocara sp (López et al., 2005).

\section{Contaminación con Toxocara sp y Eva- luación Sanitaria de Parques}

El Cuadro 2 muestra que ninguno de los factores contemplados en la ficha de evaluación del Programa de Vigilancia Sanitaria de Parques presenta asociación estadística significativa con la contaminación con Toxocara sp.

\section{Factores Asociados a la Contaminación con Toxocara sp}

El Cuadro 3 muestra que la clasificación sanitaria y el sector de ubicación de los parques no están asociados a la contaminación con Toxocara sp. El sector donde están ubicados los parques, de acuerdo al catastro municipal de Abancay, no mostró asociación significativa debido, probablemente, a que tanto el sector residencial como el comercial, se encuentran densamente poblados y el único parque ubicado en el sector agrícola es 


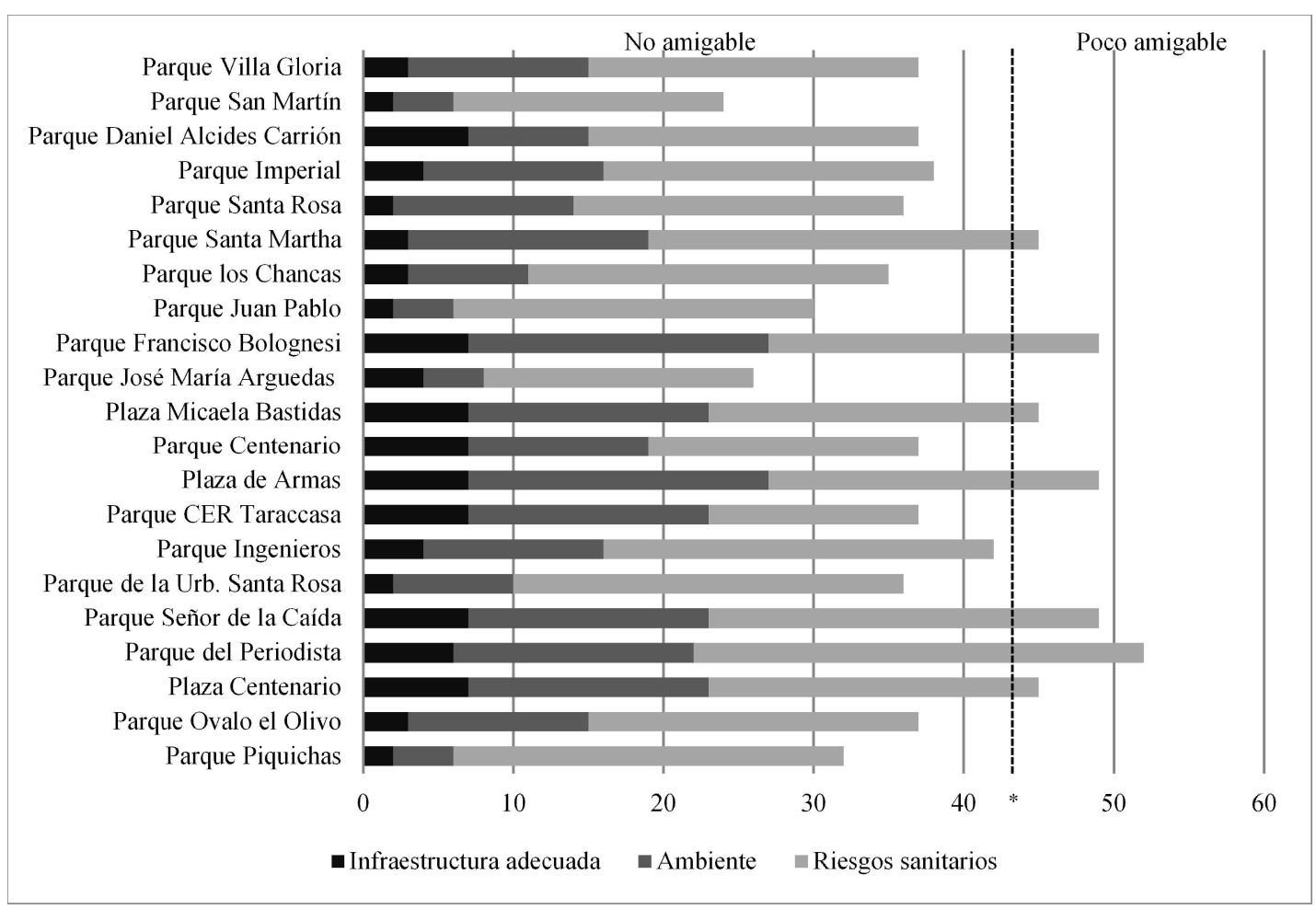

Figura 1. Evaluación sanitaria de parques en la ciudad de Abancay (2012). La línea punteada (*) limita a la izquierda a los parques que calificaron como no amigables $(\leq 42$ puntos) y a la derecha aquellos que calificaron como poco amigables ( $\geq 43$ puntos)

masivamente visitado por la existencia de un zoológico en su interior. Consecuentemente, la contaminación con huevos del parásito es mayor cuanto más densamente estén pobladas las áreas circundantes al parque (Rubel y Wisnivesky, 2005).

Los parques públicos son lugares de recreación, especialmente para los niños, por lo que representan una fuente importante de infección por Toxocara sp, debido a la aparición de huevos y larvas de este parásito zoonótico que constituye un problema de salud pública. La materia fecal de caninos es la causante de la contaminación del suelo de los parques (Díaz et al., 2015). Si bien, parásitos de los géneros Toxocara también se encuentran en las heces de gatos, estos últimos prefieren defecar en suelos arenosos donde puedan enterrar sus deyecciones, por lo que el perro doméstico sería la fuente principal de contaminación parasitaria del suelo de los parques (Armstrong et al., 2011).

La contaminación parasitaria del suelo es considerada como un indicador directo de riesgo de infección para los residentes de una región (Córdoba et al., 2002). Se ha estimado que un gramo de materia fecal de un perro cachorro puede contener hasta $15 \mathrm{mil}$ huevos de Toxocara, los cuales, al ser evacuados en la vía pública, son diseminados por el pisoteo, lluvia, viento y vectores. Sus cubiertas gruesas los hacen resistentes al frío y a cambios ambientales, por lo que pueden sobrevivir por muchos años (Romero et al., 2011). Sin embargo, estos huevos no son infectivos una vez expulsados, pues su desarrollo a estado larval toma entre 2 a 5 semanas. La infección por este parásito puede ocasionar trastornos oculares y viscerales 
Cuadro 2. Asociación entre contaminación con Toxocara sp y la evaluación sanitaria de parques en la ciudad de Abancay, Perú (2012)

\begin{tabular}{|c|c|c|c|c|}
\hline \multirow[b]{2}{*}{ Factores evaluados } & \multicolumn{2}{|c|}{ Parques } & \multirow[b]{2}{*}{$\begin{array}{c}\text { Chi } \\
\text { cuadrado }\end{array}$} & \multirow[b]{2}{*}{$\mathrm{p}$} \\
\hline & $\begin{array}{l}\text { Total } \\
\text { (n) }\end{array}$ & $\begin{array}{c}\text { Positivos a } \\
\text { Toxocara } \mathrm{sp} \\
(\%)\end{array}$ & & \\
\hline \multicolumn{5}{|l|}{ Infraestructura } \\
\hline Iluminación pública & 12 & 66.7 & 0.000 & 1.000 \\
\hline Juegos recreacionales & 6 & 83.3 & 1.050 & 0.306 \\
\hline Ausencia de veredas-senderos & 21 & 66.7 & - & - \\
\hline Ausencia de paneles educativos & 21 & 66.7 & - & - \\
\hline Bancas & 21 & 66.7 & - & - \\
\hline Ausencia de depósitos de basura & 12 & 66.7 & 0.000 & 1.000 \\
\hline \multicolumn{5}{|l|}{ Factores ambientales } \\
\hline Residuos sólidos & 15 & 66.7 & 0.000 & 1.000 \\
\hline Montículos de maleza & 8 & 62.5 & 0.101 & 0.751 \\
\hline Ausencia de tachos para heces de canes & 21 & 66.7 & - & - \\
\hline Propietario no recoge heces de canes & 21 & 66.7 & - & - \\
\hline Desagües sin protección & 5 & 40.0 & 2.100 & 0.147 \\
\hline No uso de tachos para residuos sólidos & 11 & 72.7 & 0.382 & 0.537 \\
\hline Presencia de área verde & 17 & 58.8 & 2.471 & 0.116 \\
\hline \multicolumn{5}{|l|}{ Factores de riesgo sanitario } \\
\hline Suministro inconstante de agua potable & 2 & 100.0 & 1.105 & 0.293 \\
\hline Sin suministro de agua no tratada & 21 & 66.7 & - & - \\
\hline Suministro de agua de canal de regadío & 1 & 100.0 & 0.525 & 0.469 \\
\hline No suministro de agua de desagüe & 21 & 66.7 & - & - \\
\hline Ausencia de tachos de basura con bolsa & 21 & 66.7 & - & - \\
\hline Presencia madrigueras de roedores & 1 & 100.0 & 0.525 & 0.469 \\
\hline Canes conducidos sin correa & 18 & 72.2 & 1.750 & 0.186 \\
\hline Presencia de excretas caninas & 21 & 66.7 & - & - \\
\hline Presencia de excretas humanas & 3 & 100.0 & 1.750 & 0.186 \\
\hline Venta ambulatoria de alimentos preparados & 13 & 76.9 & 1.615 & 0.204 \\
\hline Presencia de agua estancada & 2 & 100.0 & 1.105 & 0.293 \\
\hline
\end{tabular}

debido a la migración de la larva ingerida (OPS, 2003; Delgado y Rodríguez, 2009; Sariego et al., 2012). En consecuencia, el estudio demostró el riesgo potencial de toxocariosis en las personas que frecuentan los parques públicos, por lo que se necesita reforzar las medidas de control de animales callejeros y su acceso a los parques, así como la sensibilización a los propietarios para no contaminar los lugares de recreación con 
Cuadro 3. Asociación entre contaminación con Toxocara sp y la clasificación sanitaria y sector de ubicación de los parques en la ciudad de Abancay, Perú (2012)

\begin{tabular}{|c|c|c|c|c|}
\hline \multirow[b]{2}{*}{ Factores } & \multicolumn{2}{|c|}{ Parques } & \multirow[b]{2}{*}{$\begin{array}{c}\text { Chi } \\
\text { cuadrado }\end{array}$} & \multirow[b]{2}{*}{$\mathrm{p}$} \\
\hline & $\begin{array}{c}\text { Total } \\
\text { (n) }\end{array}$ & $\begin{array}{c}\text { Positivos a } \\
\text { Toxocara } \mathrm{sp} \\
(\%)\end{array}$ & & \\
\hline \multicolumn{5}{|c|}{ Clasificación sanitaria } \\
\hline No amigable & 13 & 76.9 & 1.615 & 0.204 \\
\hline Poco amigable & 8 & 50.0 & & \\
\hline \multicolumn{5}{|l|}{ Sector } \\
\hline Agrícola & 1 & 100.0 & 0.600 & 0.741 \\
\hline Residencial & 15 & 66.7 & & \\
\hline Comercial & 5 & 60.0 & & \\
\hline
\end{tabular}

excretas de sus mascotas (Armstrong et al., 2011; Díaz et al., 2015).

\section{ConClusiones}

- La prevalencia de Toxocara sp en la ciudad de Abancay es alta y se encuentra por encima del promedio nacional .

- La mayoría de los parques de la ciudad calificaron como no amigables debido a las condiciones de infraestructura, ambiente y riesgo sanitario.

- La ficha de evaluación del Programa de Vigilancia Sanitaria de Parques de la Dirección de Higiene Alimentaria y Zoonosis, MINSA, no es aplicable para estimar la contaminación con Toxocara sp en parques públicos.

\section{Literatura Citada}

1. Armstrong WA, Oberg C, Orellana JJ. 2011. Presencia de huevos de parásitos con potencial zoonótico en parques y plazas públicas de la ciudad de Temuco, Región de La Araucanía, Chile. Arch Med Vet 43: 127-134. doi: 10.4067/ S0301-732X2011000200005
2. Barbosa C. 2010. Validación parasitológica y contaminación de áreas de parques públicos de la región zona este este de la ciudad de Sao Paulo. Tesis de Maestría. Sao Paulo: Universidad de Sao Paulo. 131 p.

3. Breña JP, Hernández, Rernández $A$, Castañeda R, Espinoza I, Roldán W, Ramírez, C, Maguiña C. 2011. Toxocariosis humana en el Perú: aspectos epidemiológicos, clínicos y de laboratorio. Acta Med Per 28: 228-236.

4. Cala FA, Durán LF, Gómez C. 2010. Determinación de la presencia de estados inmaduros (huevos, larvas) de parásitos nematodos zoonóticos (Toxocara spp, Uncinaria spp y Strongyloides spp) en los parques públicos urbanos del municipio de Bucaramanga, Santander. Rev Spei Domus 6(12): 27-31.

5. Canese A, Domínguez R, Otto C, Ocampos C, Mendonca E. 2003. Huevos infectivos de Toxocara, en arenas de plazas y parques de Asunción, Paraguay. Rev Chil Pediatr 74: 611-616. doi: 10.4067/S0370-41062003000600010

6. Castillo Y, Bazán H, Alvarado D, Saez G. 2001. Estudio epidemiológico de Toxocara canis en parques recreacionales del distrito de San Juan 
de Lurigancho, Lima- Perú. Parasitol Día 25: 109-114. doi: 10.4067/S071607202001000300007

7. Cazorla DJ, Morales P, Acosta ME. 2007. Contaminación de suelos con huevos de Toxocara spp (Nematoda, Ascaridida) en parques públicos de la ciudad de Coro, Estado Falcón, Venezuela. Rev Cient (Maracaibo) 17: 117122.

8. Chávez VA, Casas AE, Serrano MM, Cajas UJ, Velarde OJ, La Rosa VV, López TJ. 2002. Riesgo de contraer enfermedades parasitarias en los parques públicos de Lima y Callao. Rev Inv Vet Perú 13: 84-91. doi: 10.15381/ rivep.v13i2.7337

9. Córdoba A, Ciarmela ML, Pezzani B, Gamboa MI, De Luca MM, Minvielle M, Basualdo JA. 2002. Presencia de parásitos intestinales en paseos públicos urbanos en La Plata Argentina. Parasitol Latinoam 57: 25-29. doi: 10.4067/S071777122002000100007

10. Cruz A, Camargo B. 2001. Glosario de términos en parasitología y ciencias afines. México DF: Plaza y Valdez. 206 p.

11. Delgado O, Rodríguez AJ. 2009. Aspectos clínico-epidemiológicos de la toxocariasis: una enfermedad desatendida en Venezuela y América Latina. Bol Mal Salud Amb 49(1): 1-33.

12. Devera R, Tutaya R, Devera R. 2015. Aislamiento de huevos y larvas de Toxocara spp y otros geohelmintos en suelos de parques de un colegio de ciudad Bolívar, estado Bolívar, Venezuela. Saber 27: 341-346.

13. Díaz AM, Pulido MO, Giraldo JC. 2015. Nematodos con potencial zoonótico en parques públicos de la ciudad de Tunja, Colombia. Salud Pública Méx 57: 170-176.

14. [DHAZ] Dirección de Higiene Alimentaria y Zoonosis. 2016. Lima: Ministerio de Salud. [Internet]. Disponible en: www.digesa.minsa.gob.pe/ DHAZ/DHAZ.asp
15. Gallardo J, Forlano M. 2015. Diagnóstico de huevos de Toxocara spp del suelo en parques y plazas públicas de la ciudad de Barquisimeto, estado Lara, Venezuela. Gaceta Cienc Vet 20(1): 4-9.

16. Gallina T, Machado MA, Laitano L, Welter E, Marreiro M, Aires ME. 2011. Presence of eggs of Toxocara spp and hookworms in a student environment in Rio Grande do Sul, Brazil. Rev Bras Parasitol Vet 20: 176-177. 10.1590/S1984-29612011000200016

17. Gutiérrez PA. 2004. Contaminación de suelos con huevos de Toxocara sp en plazas y parques públicos de la ciudad de Talca, Chile. Tesis de Tecnólogo Médico. Talca: Univ de Talca. 26 p.

18. Hernández S, Contera M, Acuña A, Elhordoy D, Vignolo J. 2003. Toxocara spp en muestras de suelo y heces de plazas de la ciudad de Montevideo. Rev Patol Trop 32(1): 95-104. doi: 10.5216/rpt.v32i1.4355

19. Huapaya P, Espinoza Y, Roldán W, Jiménez S. 2009. Toxocariosis humana: ¿problema de salud pública? An Fac Med 70: 283-290.

20. Iannacone J, Alvariño L, CárdenasCallirgos J. 2012. Contaminación de los suelos con huevos de Toxocara canis en parques públicos de Santiago de Surco, Lima, Perú, 2007-2008. Neotrop Helminthol 6(1): 97-108.

21. [INEI]. Instituto Nacional de Estadística e Informática. 2016. [Internet]. Disponible en: https://www.inei.gob.pe/

22. Laird RM, Carballo D, Reyes EM, García R, Prieto V. 2000. Toxocara sp en parques y zonas públicas de ciudad de la Habana, 1995. Rev Cubana Hig Epidemiol 38: 112-116.

23. Latorre E, Nápoles M. 2014. Estudio para determinar la contaminación con parásitos zoonóticos caninos en parques de la zona urbana del distrito metropolitano de Quito. Tesis de Médico Veterinario. Quito: Univ San Francisco de Quito. 78 p. 
24. Lettieri TM, Rossi LP, De Freitas L, Gasparin N, Piva S, Fuentefria AM. 2008. Prevalence of Toxocara canis infection in public squares of the Concordia City, Santa Catarina, Brazil. Parasitol Latinoam 63: 69-71. doi: 10.4067/S0717-77122008000100012

25. López, T, Chávez V, Casas E. 2005. Contaminación de los parques públicos de los distritos de Lima Oeste con huevos de Toxocara sp. Rev Inv Vet Perú 16: 76-81. doi: 10.15381/rivep.v16i1.1543

26. Loza A, Gonzales JL, Marin G 2006. Estudio epidemiológico de Toxocara $\mathrm{sp}$ y Ancylostoma sp en canes y paseos públicos de los distritos I al V de Santa Cruz de la Sierra. REDVET 7(9). [Internet]. Disponible en: http://www.veterinaria.org/revistas/redvet/n090906/ 090625.pdf

27. Maguiña C. 2010. Toxocariosis: un problema de salud pública en el Perú. Acta Med Per 27: 224-225.

28. Marques J, Guimarães C, Vilas A, Carnaúba P, Moraes J. 2012. Contamination of public parks and squares from Guarulhos (São Paulo State, Brazil) by Toxocara spp and Ancylostoma spp. Rev Inst Med Trop Sao Paulo 54: 267-271. doi: 10.1590/ S0036-46652012000500006

29. [OPS] Organización Panamericana de la Salud. 2003. Zoonosis y enfermedades transmisibles comunes al hombre y a los animales: parasitosis. $3^{\mathrm{a}}$ ed. Washington, DC: OPS. $413 \mathrm{p}$.

30. Paquet I, Hernandez J, Dolz G, Zuniga JJ, Schnieder T, Epe C. 2007. Prevalence of Toxocara spp, Toxascaris leonina and Ancylostomidae in public parks and beaches in different climate zones of Costa Rica. Acta Tropica 104: 30-37. doi: 10.1016/j.actatropica.2007.06.011

31. [MPA] Plan de Desarrollo Urbano Municipalidad Provincial de Abancay. 2011. Plan de Desarrollo Urbano 2011. Abancay: MPA. $481 \mathrm{p}$.

32. Polo LJ, Cortés JA, Villamil LC, Prieto E. 2007. Contaminación de los par- ques públicos de la localidad de Suba, Bogotá con nematodos zoonóticos. Rev Salud Pública 9: 550-557.

33. Romero C, García AC, Mendoza GD, Torres NC, Ramírez N. 2009. Contaminación por Toxocara spp en parques de Tulyehualco, México. Rev Cient (Maracaibo) 19: 253-256.

34. Romero C, Mendoza GD, Bustamante LP, Crosby MM, Ramírez N. 2011. Presencia y viabilidad de Toxocara spp en suelos de parques públicos, jardines de casas y heces de perros en Nezahualcóyotl, México. Rev Cient (Maracaibo) 21: 195-201.

35. Romero C, Yañez, Mendoza GD, Bustamante LP, Ramírez N. 2013. Contaminación y viabilidad de huevos de Toxocara spp en suelo y heces colectadas en parques públicos, calles y perros en Toluca, México. Rev Cient (Maracaibo) 23: 475-479.

36. Rubel D, Wisnivesky C. 2005. Magnitude and distribution of canine faecal contamination and helminth eggs in two areas of different urban structure, Greater Buenos Aires, Argentina. Vet Parasitol 133: 339-347. doi: 10.1016/ j.vetpar.2005.06.002

37. Santarém V, Franco E, Kozuki T, Fini $D$, Prestes E. 2008. Environmental contamination by Toxocara spp eggs in a rural settlement in Brazil. Rev Inst Med Trop S Paulo 50: 279-281. doi: 10.1590/ S0036-46652008000500006

38. Sariego I, Kanobana K, Rojas L, Speybroeck N, Polman K, Nuñez FA. 2012. Toxocariasis in Cuba: a literature review. PLoS Negl Trop Dis 6(2): e1382. doi: 10.1371/journal.pntd.0001382

39. Serrano M, Chávez A, Casas E. 2000. Contaminación de parques públicos del cono este con huevos de Toxocara spp. Rev Inv Vet Perú : 82-87. doi: 10.15381/rivep.v11i1.6815

40. Tinoco L, Barreras A, López G. 2007. Frecuency of Toxocara canis eggs in public parks of the urban area of Mexicali, BC, Mexico. J Anim Vet Adv 6: 430-434. 
41. Tiyo, R, Guedes, TA, Falavigna, DLM, Falavigna AL. 2008. Contaminación por Toxocara spp en parques de Tulyehualco, México. Rev Cient (Maracaibo) 19: 253-256.

42. Yapuchura JC. 2004. Contaminación de parques de la ciudad de Puno con hue vos de Toxocara spp. Tesis de Médico
Veterinario. Puno: Univ Nacional del Altiplano. $57 \mathrm{p}$.

43. Young $C$, Yauri $R$, Yance $S$, Villavicencio J, Vera K, Villegas J, Zúñiga P, Zari C, Vílchez M. 2011. Frecuencia de Toxocara sp en los parques del distrito de Breña, Lima-Perú, 2010. Rev Perú Epidemiol 15(3): 1-4. 